\title{
Transtibial ACL reconstruction technique fails to position drill tunnels anatomically in vivo 3D CT study
}

\author{
Sebastian Kopf • Brian Forsythe · Andrew K. Wong • \\ Scott Tashman · James J. Irrgang • \\ Freddie H. Fu
}

Received: 14 May 2011/Accepted: 17 December 2011/Published online: 31 December 2011

(C) The Author(s) 2011. This article is published with open access at Springerlink.com

\begin{abstract}
Purpose The purpose of this study was to visualize and quantify the positions of femoral and tibial tunnels in patients who underwent traditional transtibial single-bundle ACL reconstruction, as performed by multiple surgeons, utilizing 3D CT models, and to compare these positions to our previously reported anatomical tunnel positions.

Methods Fifty-eight knee computed tomography (CT) scans were performed on patients who underwent primary or revision transtibial single-bundle ACL reconstruction, and three-dimensional reconstructions of the $\mathrm{CT}$ scans were aligned within an anatomical coordinate system. The position of femoral tunnel aperture centers was measured with (1) the quadrant method and (2) in the anatomic posterior-to-anterior and proximal-to-distal directions. The position of tibia tunnel aperture centers were measured similarly, in the anterior-to-posterior and medial-to-lateral dimensions on the tibial plateau. Comparisons were made to previously established anatomical tunnel positions, and data were presented as "mean value \pm standard deviation (range)."

Results The location of tibial tunnels was at $48.0 \pm 5.4 \%$ (35.6-59.5\%) of the anterior-to-posterior plateau depth and at $47.9 \pm 2.9 \%(42.2-57.4 \%)$ of the medial-to-lateral plateau width. The location of femoral tunnels was at $55.8 \pm 8.0 \%(41.5-79.5 \%)$ in the anatomic posterior-to-
\end{abstract}

Electronic supplementary material The online version of this article (doi:10.1007/s00167-011-1851-z) contains supplementary material, which is available to authorized users.

S. Kopf $\cdot$ B. Forsythe $\cdot$ A. K. Wong $\cdot$ S. Tashman

J. J. Irrgang · F. H. Fu ( $₫)$

Department of Orthopaedic Surgery, University of Pittsburgh,

3471 Fifth Avenue, Pittsburgh, PA 15213, USA

e-mail: ffu@upmc.edu anterior direction and at $41.2 \pm 10.4 \%(15.1-67.4 \%)$ in the proximal-to-distal directions. Utilizing a quadrant method, femoral tunnels were positioned at $37.4 \pm 5.1 \%$ (24.9-50.6\%) from the proximal condylar surface, parallel to Blumensaat line, and at $11.0 \pm 7.3 \%(-6.0-28.7 \%)$ from the notch roof, perpendicular to Blumensaat line. In summary, tibial tunnels were positioned medial to the anatomic PL position $(p<0.001)$, and femoral tunnels were positioned anterior to both $\mathrm{AM}$ and $\mathrm{PL}$ anatomic tunnel locations $(p<0.001$ and $p<0.001)$.

Conclusion ACL reconstruction via traditional transtibial technique fails to accurately position femoral and tibial tunnels within the native ACL insertion site. To achieve anatomical graft placement, other surgical techniques should be considered.

Level of evidence IV.

Keywords ACL $\cdot$ Anatomy - Transtibial .

Anterior cruciate ligament $\cdot 3 \mathrm{D}$ CT

\section{Introduction}

It has been reported in cadaveric and clinical studies that anatomically placed tunnels better restore normal knee kinematics [24, 25]. In spite of its popularity, the ability of the transtibial technique to reliably position bone tunnels anatomically has been questioned $[4,7,18]$. Conditions such as osteoarthritis have shown to be present in a high percentage of cases using the transtibial technique, suggesting that it may not restore normal knee function or kinematics $[6,8,23]$. In addition to the initial trauma (to articular cartilage) caused by ACL injuries, it is postulated that non-anatomic bone tunnel positions may contribute to the onset of osteoarthritis. To improve clinical outcomes, 
modifications in technique have been developed to achieve a more anatomical reconstruction. These include an anatomic approach to single-bundle (SB) or double-bundle (DB) ACL reconstruction, with bone tunnels placed within the footprints of the native ACL insertions.

It is well established that tunnel position is of the utmost importance with respect to clinical outcomes [18]. However, outcome studies are difficult to compare since most of the studies in the literature fail to adequately describe tunnel position. Hence, we previously developed a standardized system for quantifying bone tunnel position in order to correlate tunnel position with clinical outcome results [9].

To better investigate bone tunnel position, 3D reconstructions of computerized tomography (CT) scans have become increasingly popular [19]. Radiographs have been the mainstay of tunnel imaging in previous studies [2, 5, 18, 26]; however, they are suboptimal for describing locations in three dimensions, whereas 3D CT models allow visualization of the entire bone as a $3 \mathrm{D}$ object. This is especially important for curved surfaces like the femoral condyle. The ability to rotate images and to isolate specific anatomical sections makes 3D CT a powerful tool for visualizing and evaluating tunnel position after ACL reconstruction (Figs. 1, 2, and 3).

Previously, we evaluated positions of transtibial tunnel apertures in a selected patient cohort [16]. In the current study, 3D CT scans were additionally evaluated in a broader cohort of patients, including those who were symptomatic (with pain and/or instability) following primary and revision ACL reconstruction. An advantage of the current study is that 3D CT images are made available for all cases in the Supplementary material in Appendix, so that surgeons are provided with a reference with which to compare their own patients' tunnel positions.

Thus, the purpose of this study was to visualize and quantify the positions of femoral and tibial tunnels in patients who underwent traditional transtibial single-bundle ACL reconstruction, as performed by multiple surgeons, utilizing 3D CT models, and to compare these positions to our previously reported anatomical tunnel positions [9].

\section{Materials and methods}

In total, 58 knee CT scans were performed on 53 patients [33 males, 20 females, mean age 37.0 ( \pm 10.2 years (17.1-59.6)] who had undergone arthroscopic transtibial single-bundle ACL reconstruction. Five patients had ACL reconstructions performed bilaterally; both knees of each patient were included, and one patient with bilateral transtibial ACL reconstruction had two femoral tunnels that were included.
Only one tibia was excluded from the study for lack of a clearly identifiable tunnel aperture on $3 \mathrm{D}$ reconstruction of the CT scan. Twenty-six surgeons performed the surgeries between 1989 and 2007. Eleven CT scans were performed in asymptomatic subjects in association with a routine follow-up for a prospective study, and twenty-one CT scans were performed on patients in our clinic for various clinical reasons other than increased knee laxity and ACL reinjury. The mean time from surgery to the CT scan was $6.7 \pm 5.8$ years. A standard CT scan protocol was used. IRB approval was obtained from the two institutions where CT scans were performed.

Three-dimensional reconstruction of CT scans

3D CT model reconstructions and measurements were all performed with the detailed methods described in our prior cadaveric study [9], which evaluated tunnel locations of anatomic double-bundle ACL reconstruction. An abridged version of the methods is provided in the current article.

3D CT models were reconstructed from axial CT scan slices using Mimics software (Materialise, Leuven, Belgium) and exported as pointcloud data into Geomagic software (Research Triangle Park, North Carolina, USA). 3D surface models were created and coregistered with gender-specific models pre-aligned in an anatomical coordinate system.

In order to maintain consistency throughout this article, tunnel positions are described relative to anatomic orientation of the knee in full extension (Fig. 1a). Thus, position will be described using the terms anterior, posterior, proximal, distal, medial, and lateral [corresponding to superior, inferior, deep, shallow, medial, lateral, respectively, and in common arthroscopic terminology (Fig. 1b)].

Utilizing a true top-down view (perpendicular to the proximal/distal axis of the tibia), the anterior-to-posterior (A-P) and medial-to-lateral (M-L) tunnel positions were measured and calculated as percentages of the maximum A-P and M-L dimensions of the tibial plateau.

On the femoral side, two different techniques were used to measure the tunnel aperture locations. First, a novel measurement system was used within the anatomical coordinate system defined relative to structures visualized arthroscopically. Second, the quadrant method by Bernard and Hertel et al. [5] was utilized.

\section{Anatomic coordinate axes method}

A true medial view on the medial wall of the lateral condyle (perpendicular to the medial/lateral femoral axis) at $90^{\circ}$ flexion was established. Tunnel positions were measured in the posterior-to-anterior (P-A) and proximal-todistal (P-D) directions using ImageJ software (NIH ImageJ, 

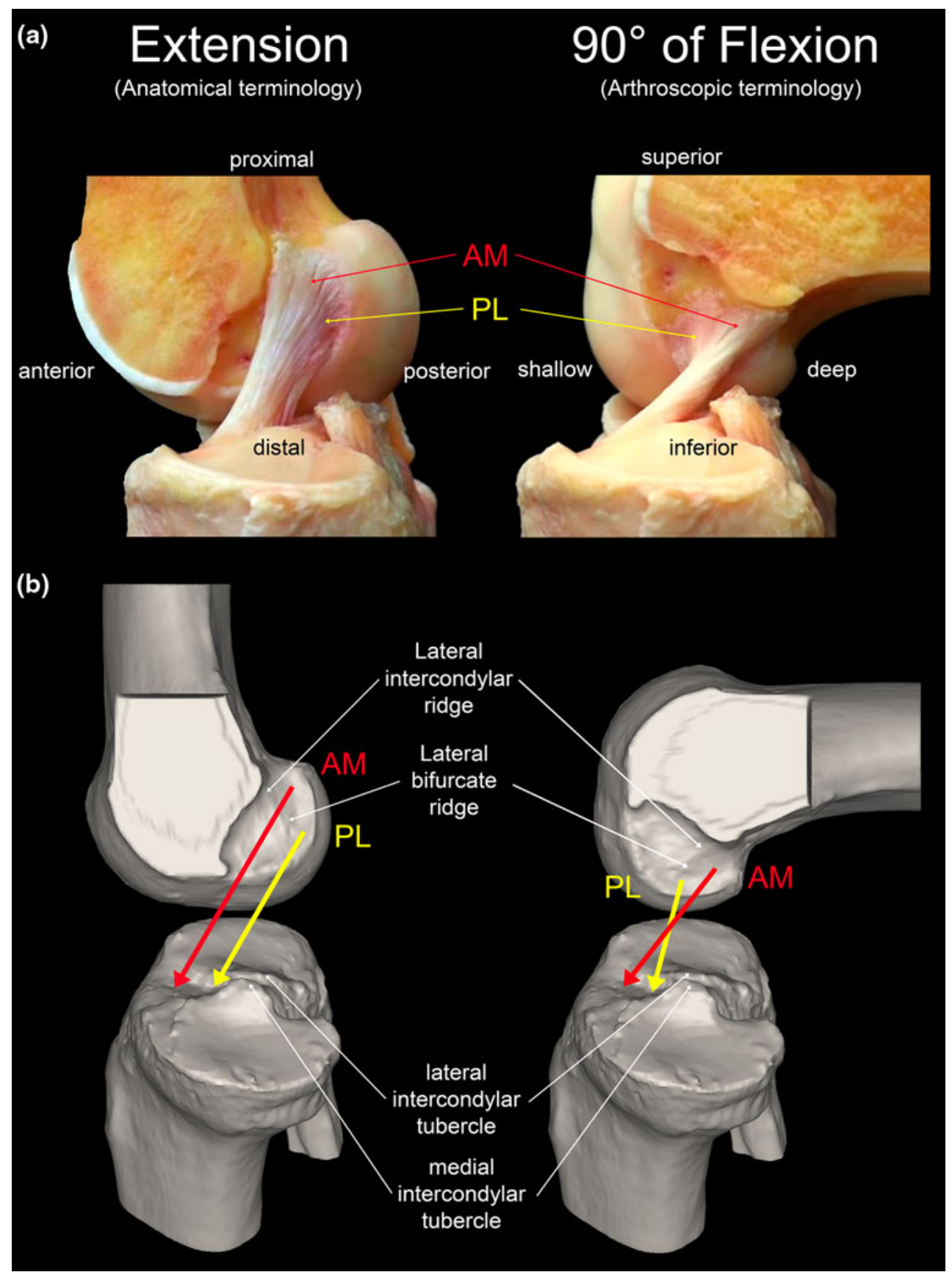

Fig. 1 On the cadaveric and 3D CT models shown here, the orientation of the AM and PL bundle insertions is well demonstrated. When the knee is in extension $\left(0^{\circ}\right)$, the ACL insertion is nearly vertical with the AM insertion proximal to the PL insertion. When the knee is in flexion $\left(90^{\circ}\right)$, the ACL insertion is nearly horizontal. The

$\mathrm{NIH}$, Bethesda, MD, USA). The measurements were made relative to arthroscopically relevant structures (Fig. 2). P-A positions were calculated as percentages from the posterior border of the medial wall of the lateral condyle to the most anterior point of the notch. $P-D$ positions were calculated as percentages from the proximal border of the notch to the distal point of the notch roof. proximal-distal orientation of the insertion remains the same (AM insertion is proximal to the PL insertion); however, when viewed arthroscopically with the knee at $90^{\circ}$ of flexion, the AM insertion is described as being "behind" or "deep to" the PL insertion

\section{Quadrant method}

Using the same view of the femur as the Anatomic Coordinate Axes Method above, a $4 \times 4$ grid system was applied parallel/perpendicular to Blumensaat line, and measurements were performed as previously described [5]. 


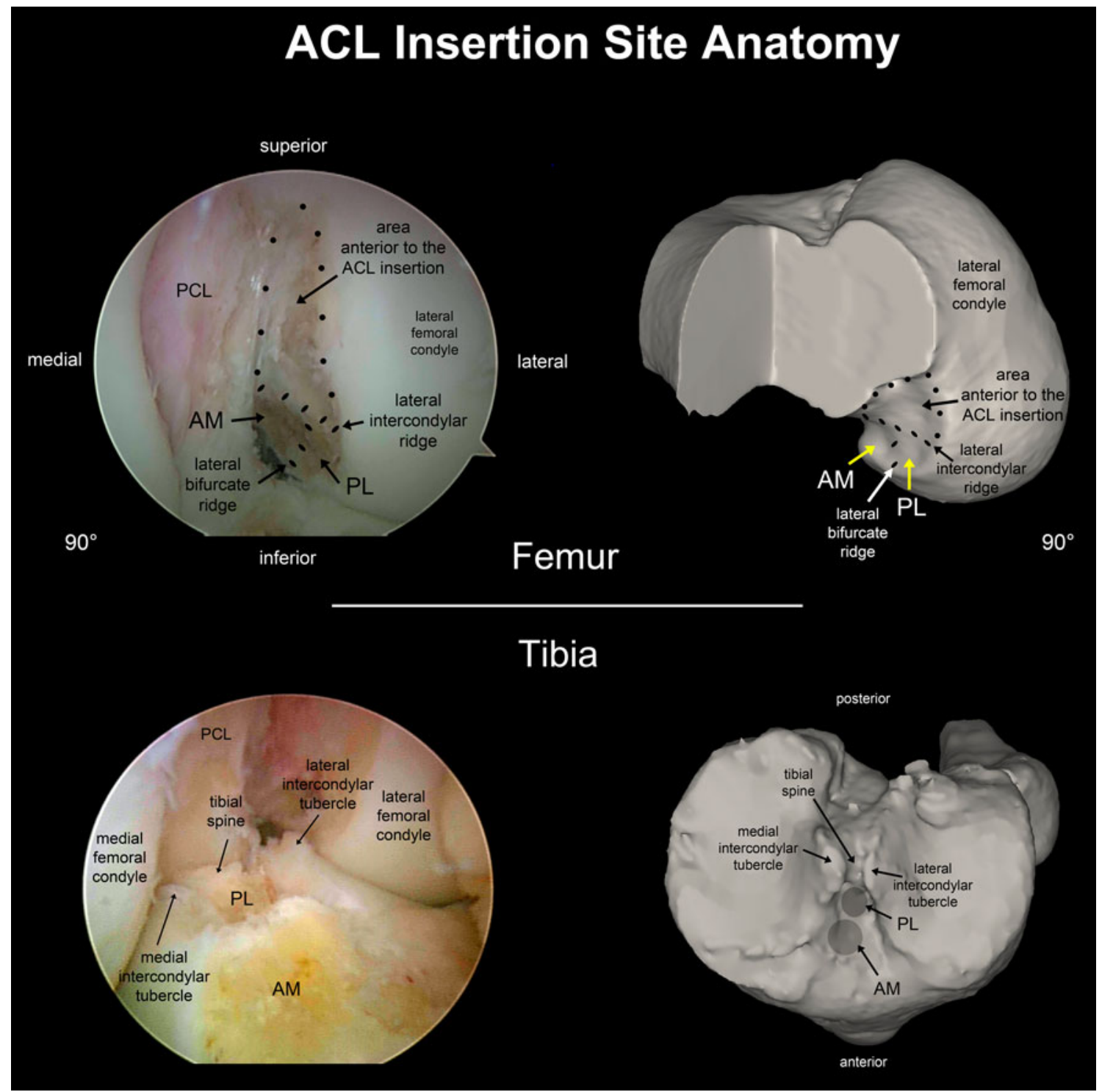

Fig. 2 On the femur, the lateral intercondylar and bifurcate ridges are visualized arthroscopically and by $3 \mathrm{D}$ CT. The area anterior the AM and PL insertions is outlined (circular dots) to demonstrate that the entire femoral ACL insertion lies posterior to the lateral intercondylar

\section{Statistical analysis}

Data were presented as "mean value \pm standard deviation (range)." Anatomic comparison data were taken from our previous cadaver study. Independent t-tests were performed to compare: (1) transtibial single-bundle tunnel position versus anatomic AM tunnel position, and (2) transtibial single-bundle tunnel position versus anatomic PL tunnel position. To account for the two comparisons of the femoral and tibial tunnels, the significance level was set at $p<0.025$. SPSS software (SPSS Inc., IL, USA) was used for all statistical analyses. ridge on the medial wall of the lateral femoral condyle. On the tibia, the PL insertion lies anterior to the tibial spine, and the AM and PL insertions lie between the medial and lateral intercondylar tubercles

\section{Results}

3D CT model images of each patient are provided online in the Supplementary material in Appendix of this article.

With respect to tibia tunnel positions in the A-P dimension, the transtibial tunnels $(48.0 \pm 5.4 \%)$ were not significantly different than the anatomic PL tunnel positions $(46.4 \pm 3.7 \%, p=0.48)$; however, they were significantly posterior to the anatomic AM tunnel positions $(25.0 \pm 2.8 \%, p<0.001)$. In the M-L dimension, the positions of the transtibial tunnels $(47.9 \pm 2.9 \%)$ were more medial than the positions of the anatomic AM tunnels 
$(50.5 \pm 4.2 \%, p=0.03)$ and more medial than the positions of the anatomic PL tunnels $(52.4 \pm 2.5 \%$, $p<0.001)$.

Femoral anatomical coordinate axes measurements

In the P-A dimension, the transtibial femoral tunnels $(55.8 \pm 8.0 \%)$ were positioned anterior (or "high") in comparison to the anatomic AM and PL tunnels $(23.1 \pm 6.1 \%, p<0.001$ and $15.3 \pm 4.8 \%, p<0.001$, respectively). In the $\mathrm{P}-\mathrm{D}$ dimension, the transtibial femoral tunnels $(41.2 \pm 10.4 \%)$ were positioned distal relative to the anatomic AM tunnels $(28.2 \pm 5.4 \%, p=0.001)$ and proximal to the PL tunnels $(58.1 \pm 7.1, p<0.001)$.

\section{Quadrant method measurements}

Along line " $h$ ", perpendicular to Blumensaat line, the transtibial tunnels $(11.0 \pm 7.3 \%)$ were positioned significantly closer (or more anterior) to Blumensaat line compared to the anatomic AM and PL tunnels (33.2 $\pm 5.6 \%$, $p<0.001$ and $55.3 \pm 5.3 \%, p<0.001$, respectively). Along line " $t$ ", parallel to Blumensaat line, the position of the transtibial tunnels $(37.4 \pm 5.1 \%)$ relative to the anatomic AM tunnels was significantly different $(21.7 \pm$ $2.5 \%, p<0.001)$; furthermore, the transtibial tunnels were in closer proximity to the anatomic PL tunnels $(35.1 \pm$ $3.5 \%, p=0.21)$. Within the $4 \times 4$ grid, the tunnels were located in $1 \mathrm{~b}$ (56 tunnels), 1c (1 tunnel), 1a (1 tunnel), and 2b (1 tunnel).

\section{Discussion}

This study demonstrates that the traditional transtibial technique for arthroscopic ACL reconstruction results in bone tunnel apertures that are positioned non-anatomically: Femoral tunnels were anterior ("high") to the anatomic AM and PL tunnels, and tibial tunnels were medial to the anatomic PL tunnels. The results of this study are comparable to cadaveric studies of tunnel position of traditional transtibial ACL reconstruction surgery, in which tunnels were drilled arthroscopically or via an open approach and evaluated post-operatively by dissection [3, 10, 15, 21].

These findings are consistent with previous reports utilizing 3D methodologies. An in vivo study evaluated femoral tunnel position after transtibial drilling by MRI and found that the transtibial technique results in nonanatomical tunnel placement [1]. Although their results were similar to ours, the authors did not characterize tibial tunnel positions, the sample size was small $(n=8)$, and MRI was utilized to evaluate bone tunnel positions instead of CT scan, which is the gold standard to evaluate bony morphologic structures. These results were confirmed by a recent in vivo CT study in twenty patients [20]. Utilizing a transtibial technique with a tibial guide set between 60 and 65 degrees in the coronal plane relative to the medial tibial plateau (Howell 65 ${ }^{\circ}$ Tibial Guide; Biomet), femoral tunnels could not be placed into the anatomic center of the femoral ACL insertion site. Furthermore, it seems that a non-anatomic posterior position of the tibial tunnel was employed according to the picture demonstrating their technique, although the tibial tunnel position was not further evaluated [20].

There are also previous reports claiming that anatomic femoral insertion sites can be achieved with transtibial drilling [11, 12]. A retrospective radiographic study of post-operative transtibial ACL reconstruction patients determined that tibial tunnels were positioned posteriorly along the tibial plateau, and that femoral tunnels were positioned anatomically in reference to measurements along the Blumensaat line [11]. However, use of onedimensional measures along the Blumensaat line alone is insufficient to ascertain the anatomical location of the femoral ACL insertion. One-dimensional radiographic measurements should not be utilized to define tunnel locations within the 3D notch, without correlation by arthroscopic images, 3D CT imaging, or gross dissection. A cadaveric study found that while it was possible to achieve anatomic placement with transtibial drilling, transtibially established femoral tunnels were associated with short tibial bone tunnels and anterior tibial tunnel entrances too close to the joint line [12]. They, therefore, suggested an accessory medial portal approach for femoral tunnel drilling.

The outcomes reported in the present study are bolstered by the fact that the ACL reconstructions were performed by different surgeons from multiple institutions: Tunnel positions drilled using the same transtibial SB technique were consistently placed non-anatomically. We consider this to be a relative strength; had one surgeon performed all of the surgeries, bias would be increased and generalizability decreased.

Prior to the emergence of arthroscopic procedures, ACL reconstruction was predominantly performed as an open two-incision surgery. The likelihood that both the bone tunnels, as well as the ACL itself, were anatomically reconstructed was actually quite high, given the advantage of direct visualization. During the 1980s, as the paradigm of ACL surgery shifted to arthroscopic single-incision techniques; transtibial techniques (Fig. 3) were adopted to provide more efficient operations, faster recovery times, and minimal trauma to the patient. However, as suggested by the current study, the traditional transtibial technique appears to limit the surgeon's view of the anatomy, making it more difficult to recreate the ACL's native femoral 
insertion site. Furthermore, the tibial tunnel trajectory may increase the risk of non-physiological impingement. This concern over impingement leads to posterior placement of the tibial tunnel and may result in a mismatched graft, positioned from a tibial PL position to a femoral anterior ("high") AM position (Fig. 3). The data indicate that traditionally drilled tibial tunnels were positioned at the anatomic PL position in the A-P direction, and transtibially drilled femoral tunnels were positioned in an anterior (or "high") position compared to the anatomic AM and PL tunnel positions in the P-A direction. Concerns over impingement have also led to widespread use of notchplasties in conjunction with transtibial-drilled tunnels, adding additional trauma to an already compromised joint. It is our contention that anatomic positioning of the ACL will prevent the occurrence of pathological impingement, eliminating the need for notchplasty and its associated trauma.

Misplaced tunnels have become an increasing concern, due largely to growing evidence that traditional transtibial ACL reconstruction does not restore normal dynamic knee function [22, 23]. A long-term study of 200 patients also showed that more posteriorly placed femoral bone tunnels, closer to the native insertion site, resulted in better rotational control and less instability of the knee [18]. These concerns have motivated the current trend toward anatomical tunnel placement, inspired by cadaver studies demonstrating that the anatomical approach more closely restores the original ACL anatomy and knee kinematics $[14,17,24,25]$.

An important aspect of this study is the use of full 3D analysis techniques for characterizing tunnel position. Other traditional methods of measuring tunnel position, such as the Amis and Jakob line for tibial bone tunnel position, utilize plain radiographic images [2]. Unfortunately, because plain radiographs are merely $2 \mathrm{D}$ projected images of 3D structures, alignment of the bone within the imaging plane affects accurate measurements, and, thus, significant errors may be introduced in measured tunnel positions. Thus, radiographic images may not be adequate to describe, visualize, or define the 3D location of bone tunnels drilled during ACL reconstruction surgery. 3D CT models enable visualization of bone in its entirety without the 2D restrictions of plain radiographs. Subtle topographical features, which were previously seen only during arthroscopy or gross dissection, are easily discernable on 3D CT models (Figs. 1 and 2). Additionally, for improved visualization of these anatomical landmarks, selected sections may be removed, and the 3D models may be rotated, for instance, as seen during surgery. In the current study,

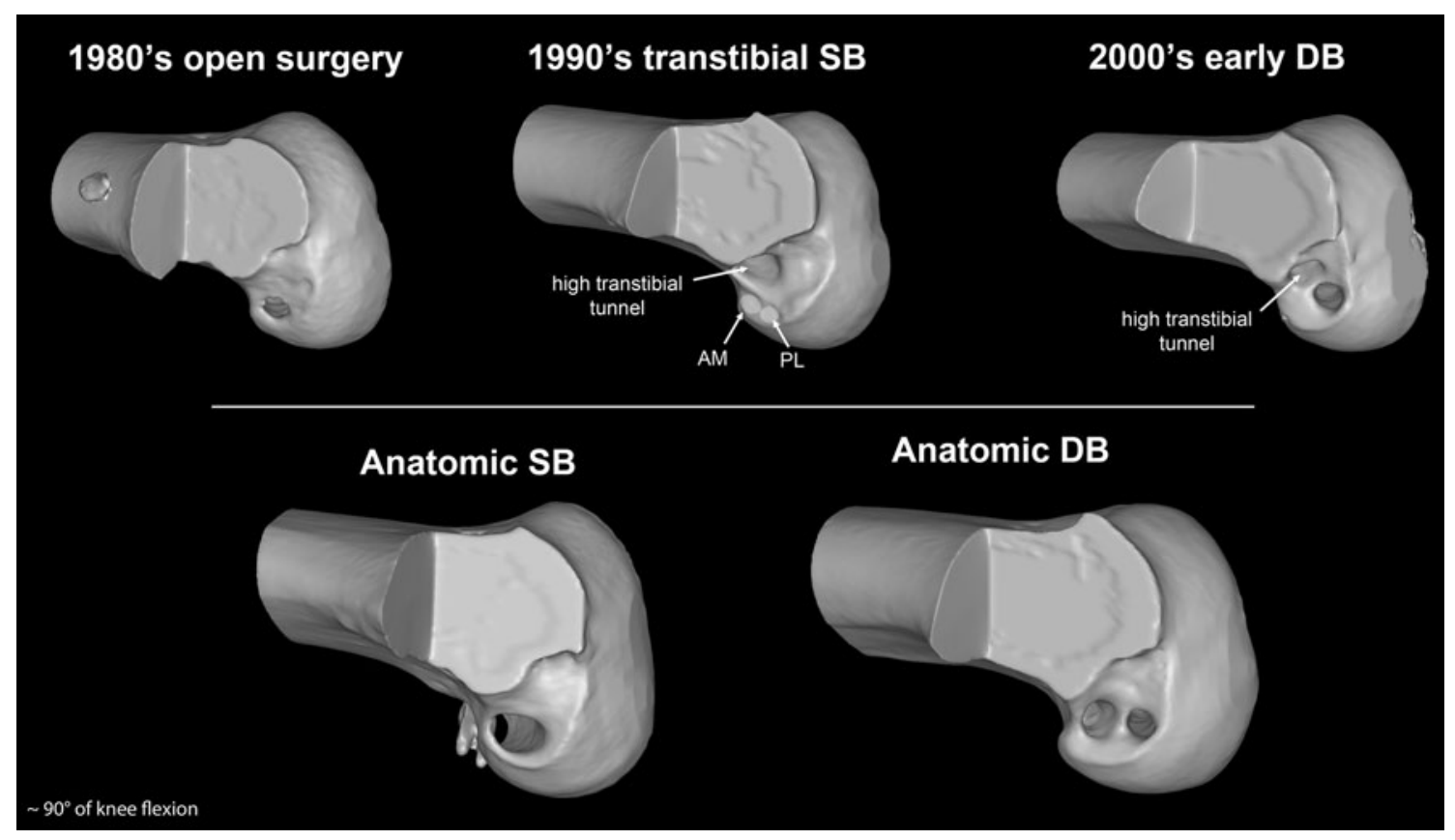

Fig. 3 ACL reconstruction tunnel placement has evolved circuitously over the previous three decades. In the 1980s, ACL bone tunnels were placed anatomically utilizing outside-in jigs and mini-open or open techniques. In the 1990s, arthroscopic assisted transtibial techniques were popularized, but femoral tunnels were placed in a nonanatomical anterior ("high AM") position. In the early 2000s, double-bundle techniques were introduced to our institution. The PL bone tunnel was drilled anatomically via the accessory medial portal, but the femoral AM bone tunnel was drilled via the tibial AM tunnel resulting in a non-anatomic anterior ("high AM") position once again. Our anatomical knowledge has further improved since that time, and we have subsequently placed our bone tunnels anatomically into the native ACL insertions 
the medial condyles were removed for better visualization of the medial wall of the lateral condyle, and the 3D bone models were aligned with an anatomically defined coordinate system, in order to make measurements independent of limb orientation during imaging.

A limitation of this study may be that a subset of patients presented to the clinic for a variety of clinical reasons (such as meniscal injury and patellofemoral pain), not necessarily related to their ACL surgery. If there exists a relationship between tunnel position and outcome, it is possible that this might introduce a selection bias in favor of non-anatomic tunnel positions. Importantly, patients with reinjury (partial or complete tear) and/or increased knee laxity (KT-2000 $>3 \mathrm{~mm}$, positive Lachman and/or positive anterior drawer tests) of the reconstructed ACL were excluded from the study. Another limitation is the inability to compare femoral and tibial tunnel aperture center positions drilled transtibially, to a patient's unique, individual anatomic ACL origin and insertion. No CT scans were obtained on normal contralateral knees of the patients in this study. Instead, comparisons were made between the transtibial patient data and the anatomic cadaveric data. To account for variations in knee sizes, measurements were normalized to known dimensions within the knee, and the results were expressed as percentage.

Another limitation is that it was not possible to control for possible tunnel aperture migration, resulting from tunnel widening. However, a prospective CT study by Iorio et al. [13] described tunnel widening of less than $1 \mathrm{~mm}$ between $\mathrm{CT}$ scans taken between post-operative day one and at 10 months post-operatively (range 9-11 months post-operatively). Most of the tunnel widening occurred during the first 6 months post-operatively. The direction of tunnel widening, however, was not investigated in this study or in others reported in the literature. We believe that it is unlikely that tunnel aperture centers migrated from anatomic to non-anatomic positions as a result of tunnel widening.

\section{Conclusion}

ACL reconstruction via traditional transtibial technique fails to accurately position femoral and tibial tunnels within the native ACL insertion site. Thus, surgeons aiming for anatomical ACL graft placement should consider other surgical techniques. This study provided analysis by 3D CT models, a reproducible and precise method of measuring and demonstrating bone tunnel position.

Acknowledgments This work was partially supported by a grant from the National Institutes of Health, NIAMS AR46387.

Conflict of interest None.
Open Access This article is distributed under the terms of the Creative Commons Attribution Noncommercial License which permits any noncommercial use, distribution, and reproduction in any medium, provided the original author(s) and source are credited.

\section{References}

1. Abebe ES, Moorman CT, Dziedzic TS, Spritzer CE, Cothran RL, Taylor DC, Garrett WE, Defrate LE (2009) Femoral tunnel placement during anterior cruciate ligament reconstruction: an in vivo imaging analysis comparing transtibial and 2-incision tibial tunnel-independent techniques. Am J Sports Med 37:1904-1911

2. Amis AA, Jakob RP (1998) Anterior cruciate ligament graft positioning, tensioning and twisting. Knee Surg Sports Traumatol Arthrosc 6(Suppl 1):S2-S12

3. Arnold MP, Kooloos J, van Kampen A (2001) Single-incision technique misses the anatomical femoral anterior cruciate ligament insertion: a cadaver study. Knee Surg Sports Traumatol Arthrosc 9:194-199

4. Bedi A, Musahl V, Steuber V, Kendoff D, Choi D, Allen AA, Pearle AD, Altchek DW (2011) Transtibial versus anteromedial portal reaming in anterior cruciate ligament reconstruction: an anatomic and biomechanical evaluation of surgical technique. Arthroscopy 27:380-390

5. Bernard M, Hertel P, Hornung H, Cierpinski T (1997) Femoral insertion of the ACL. Radiographic quadrant method. Am J Knee Surg 10:14-21 Discussion 21-2

6. Biau DJ, Tournoux C, Katsahian S, Schranz P, Nizard R (2007) ACL reconstruction: a meta-analysis of functional scores. Clin Orthop Relat Res 458:180-187

7. Dargel J, Schmidt-Wiethoff R, Fischer S, Mader K, Koebke J, Schneider T (2009) Femoral bone tunnel placement using the transtibial tunnel or the anteromedial portal in ACL reconstruction: a radiographic evaluation. Knee Surg Sports Traumatol Arthrosc 17:220-227

8. Fithian DC, Paxton EW, Stone ML, Luetzow WF, Csintalan RP, Phelan D, Daniel DM (2005) Prospective trial of a treatment algorithm for the management of the anterior cruciate ligamentinjured knee. Am J Sports Med 33:335-346

9. Forsythe B, Kopf S, Wong AK, Martins CAQ, Anderst W, Tashman S, Fu FH (2010) The location of femoral and tibial tunnels in anatomic double-bundle anterior cruciate ligament reconstruction analyzed by three-dimensional computed tomography models. J Bone Jt Surg Am 92:1418-1426

10. Gavriilidis I, Motsis EK, Pakos EE, Georgoulis AD, Mitsionis G, Xenakis TA (2008) Transtibial versus anteromedial portal of the femoral tunnel in ACL reconstruction: a cadaveric study. Knee 15:364-367

11. Gougoulias N, Khanna A, Griffiths D, Maffulli N (2008) ACL reconstruction: can the transtibial technique achieve optimal tunnel positioning? A radiographic study. Knee 15:486-490

12. Heming JF, Rand J, Steiner ME (2007) Anatomical limitations of transtibial drilling in anterior cruciate ligament reconstruction. Am J Sports Med 35:1708-1715

13. Iorio R, Vadalà A, Argento G, Di Sanzo V, Ferretti A (2007) Bone tunnel enlargement after ACL reconstruction using autologous hamstring tendons: a CT study. Int Orthop 31:49-55

14. Jepsen CF, Lundberg-Jensen AK, Faunoe $P$ (2007) Does the position of the femoral tunnel affect the laxity or clinical outcome of the anterior cruciate ligament-reconstructed knee? A clinical, prospective, randomized, double-blind study. Arthroscopy 23:1326-1333

15. Kaseta MK, DeFrate LE, Charnock BL, Sullivan RT, Garrett WE (2008) Reconstruction technique affects femoral tunnel placement in ACL reconstruction. Clin Orthop Relat Res 466:1467-1474 
16. Kopf S, Forsythe B, Wong AK, Tashman S, Anderst W, Irrgang JJ, Fu FH (2010) Nonanatomic tunnel position in traditional transtibial single-bundle anterior cruciate ligament reconstruction evaluated by three-dimensional computed tomography. J Bone Jt Surg Am 92:1427-1431

17. Musahl V, Plakseychuk A, VanScyoc A, Sasaki T, Debski RE, McMahon PJ, Fu FH (2005) Varying femoral tunnels between the anatomical footprint and isometric positions: effect on kinematics of the anterior cruciate ligament-reconstructed knee. Am J Sports Med 33:712-718

18. Pinczewski LA, Salmon LJ, Jackson WF, von Bormann RB, Haslam PG, Tashiro S (2008) Radiological landmarks for placement of the tunnels in single-bundle reconstruction of the anterior cruciate ligament. J Bone Jt Surg Br 90:172-179

19. Purnell ML, Larson AI, Clancy W (2008) Anterior cruciate ligament insertions on the tibia and femur and their relationships to critical bony landmarks using high-resolution volume-rendering computed tomography. Am J Sports Med 36:2083-2090

20. Silva A, Sampaio R, Pinto E (2010) Placement of femoral tunnel between the AM and PL bundles using a transtibial technique in single-bundle ACL reconstruction. Knee Surg Sports Traumatol Arthrosc 18:1245-1251
21. Steiner ME, Battaglia TC, Heming JF, Rand JD, Festa A, Baria M (2009) Independent drilling outperforms conventional transtibial drilling in anterior cruciate ligament reconstruction. Am J Sports Med 37:1912-1919

22. Tashman S, Collon D, Anderson K, Kolowich P, Anderst W (2004) Abnormal rotational knee motion during running after anterior cruciate ligament reconstruction. Am J Sports Med 32:975-983

23. Tashman S, Kolowich P, Collon D, Anderson K, Anderst W (2007) Dynamic function of the ACL-reconstructed knee during running. Clin Orthop Relat Res 454:66-73

24. Yagi M, Kuroda R, Nagamune K, Yoshiya S, Kurosaka M (2007) Double-bundle ACL reconstruction can improve rotational stability. Clin Orthop Relat Res 454:100-107

25. Yagi M, Wong EK, Kanamori A, Debski RE, Fu FH, Woo SL (2002) Biomechanical analysis of an anatomic anterior cruciate ligament reconstruction. Am J Sports Med 30:660-666

26. Zantop T, Diermann N, Schumacher T, Schanz S, Fu FH, Petersen W (2008) Anatomical and nonanatomical double-bundle anterior cruciate ligament reconstruction: importance of femoral tunnel location on knee kinematics. Am J Sports Med 36:678-685 